\title{
16
}

\section{The Future for Land Use Mapping: National E-Infrastructure, Modelling Analytics, Synthesis and Securing Institutional Capacity}

\section{Tim Clancy, Brett A. Bryan and Siddeswara M. Guru}

\section{Key Points}

- Land use and land use change are central to our understanding of human impacts on the environment; they underpin policy and programs to support productivity improvements, protection and land development.

- Increased demands for information and improved technologies provide a driver to improve our ability to characterise, track and model future trends in land use.

- Improved technologies, applications and synthetic approaches, such as increased analytical power through harnessing cloud computing and high-performance processing, provide significant opportunities.

- Some of these recent technologies, both within and outside the land use mapping context, are presented to map a vision for the future. 
- The establishment of a land use and land resources centre-a virtual organisation operating on a collaborative basis and drawing on the expertise of Australian agencies and research institutions with allied interests and objectives-would be an excellent way of securing the institutional capabilities to deliver on this vision.

\section{Introduction}

Land use and land use change are central to our understanding of human impacts on the environment (e.g. Foley et al., 2005); they underpin policy and programs to support productivity improvements, protection and use of our natural resources and land development (Clancy \& Lesslie, 2013). Increased demands for information and improved technology provide both a driver of change to existing approaches and an opportunity to improve our ability to characterise, track and model future trends in land use (Bryan, 2013). In this chapter, we explore some of these opportunities, focusing on current advances in e-infrastructure and examples of their application. A goal of this paper is to look at ways to address one of Rob Lesslie's key concerns: the ad hoc nature of both the funding and delivery of crucial national and regional land use products. Some of these recent technologies, both within and outside the land use mapping context, are presented to map a vision for the future, including the establishment of a centre for land use and land resources.

\section{Current and Future Drivers}

Land use decisions have a direct impact on our food production systems, natural environment and communities, and are central to many current debates (Lesslie \& Mewett, 2013). It is relatively easy to identify a broad range of drivers, both internationally and domestically, for access to highquality land use information. High quality is defined as:

- accurate representation of on-ground land use

- precise measures of trends in land use change

- sound future scenarios

- management-appropriate scales. 
Issues such as food security, agriculture productivity improvements, (ecologically) sustainable development, biosecurity and disasterdegradation mitigation are dependent on understanding land use. These issues provide important contextual information for the scientific and policy analyses undertaken by international bodies, such as the United Nations Framework Convention on Climate Change, Convention on Biological Diversity and United Nations Convention to Combat Desertification. At the national level, elements of these international drivers are relevant; so too are specific priorities, such as current aspirations to foster development of northern Australia, land-based carbon capture programs and the management of nutrient run-off onto the Great Barrier Reef.

This diversity of drivers is both a strength and a weakness in the development of robust, consistent and high-quality land use information. The strength is in the value placed on the information provided; the weakness is that there is no single issue that drives investment, nor a single agency or single level of government with ultimate responsibility.

\section{Increasing Computational Demands from Land Science}

The drivers of and solutions to climate change, food and energy security, natural resource management and biodiversity conservation all reside within extremely complex socio-ecological systems that demand the integrated assessment and modelling of multidisciplinary 'big' data (Bryan, 2013).

Increasingly complex and interconnected problems confronting land use scientists, managers and policymakers in the land system require new technological approaches. The solution to complex global challenges requires information on the management of land use at high spatial and temporal resolutions over continental or global extents. However, until recently, computing capacity was a barrier to the sort of large-scale, highresolution modelling required (Zhao et al., 2015). Bryan, Crossman, King and Meyer (2011) provided examples of the type of complex analyses required in dealing with issues of sustainable agriculture. Often, we are interested in the analysis of potential future landscapes, which require multiple objectives, multiple scenarios, complex prioritisation and 
quantification of a range of impacts. Even when dealing with constrained geographic areas, such analyses require large amounts of input data and significant computing power.

\section{High-Performance Computing}

The computational demands of integrated modelling across space and time (hindcasting and forecasting), as well as the realities of the complex socialecological systems required to address global environmental challenges, are unlikely to be met by traditional geographic information system (GIS) tools that are largely constrained to the desktop or local server. Bryan (2013) evaluated the potential of a range of high-performance computing (HPC) hardware and software tools to overcome these computational barriers and found clear potential for spectacular gains in performance. He conducted four realistic simulation experiments using:

a. Arc Macro Language (AML) GIS script on a single central processing unit (CPU)

b. Python/NumPy on 1-256 CPU cores

c. Python/NumPy on 1-64 graphics processing units (GPUs) with high-level PyCUDA abstraction (GPUArray)

d. Python/NumPy on 1-64 GPUs with low-level PyCUDA abstraction (ElementwiseKernel).

The gains in performance from the GIS implementation, which effectively took 15.5 weeks to run, were marked, with speed gains of $59 \times$ for scenario b) compared to a). More impressively, there was a $4,881 \times$ increase using ElementwiseKernel c) compared to a) with, at best, the ElementwiseKernel module in parallel over 64 GPUs achieving a speed-up of $>60,000 \times$ d) compared to a).

Open source tools, such as Python, applied across a spectrum of HPC resources, offer transformational and accessible performance improvements for integrated assessment and modelling. By reducing the computational barrier, HPC can lead to a step change in modelling sophistication, including the better representation of uncertainty and, perhaps, new modelling paradigms (Bryan, 2013). 
There are currently major costs with migration to new hardware and software environments in the HPC environment; however, as Bryan (2013) pointed out, if researchers can be freed of the computational constraints with access to HPC, they can develop new approaches to addressing global environmental challenges, which augurs well for an exciting future.

\section{Use of a Scientific Workflow Approach}

A driver of e-infrastructure development is the move from data storage and computer-focused analyses (generally limited by local infrastructure) to a focus on methodologies as tools and data, where these are brought together and cloud computing and storage systems are fully harnessed (Francis, 2015). Scientific workflow technology has become popular by providing a high-level environment that can automate, manage and execute various steps in scientific research, while also having the ability to store and track provenance information. Scientific workflows provide a powerful unifying platform that allows scientists to arbitrarily build complicated applications by combining predefined components that may be implemented in different programming languages (Ludäscher et al., 2006). Once the workflow is built, it can be re-used and re-executed with minimal effort. These intrinsic capabilities of a workflow system with provenance tracking functionality would improve the reproducibility of experiments and encourage the sharing of experimental processes and results. Workflow systems offer a broad range of components that perform tasks, ranging from acquiring data from sensors, querying databases, datamining and visualisation through to execution of arbitrary applications.

The Collaborative Environment for Ecosystem Science Research and Analysis (CoESRA) system developed by the Terrestrial Ecosystem Research Network is a web-enabled, virtual desktop environment, running on a cloud infrastructure. This is an example of where a workflow approach (effectively an analysis tool) can be integrated into national computer and storage infrastructure that addresses the barriers to entry to HPC and also provides a scalable and flexible virtual laboratory environment. ${ }^{1}$ Users can access a virtual desktop environment to build, execute and share

1 This system is available online from www.coesra.org.au 
workflow-based scientific analyses and syntheses activities. The system supports Australian Access Federation as a login mechanism (Guru et al., 2015).

The CoESRA platform is built on Queensland Research and Innovation Services Cloud (QRISCloud) infrastructure, which enables access to data sources that are available from web services, large storage, distributed computing infrastructure and analysis tools to implement cases from the ecosystem science community. Using CoESRA, scientists can make their analyses repeatable and sharable, which may improve the uptake of scientific outcomes (Guru et al., 2015).

Several innovative concepts were developed as part of the system to reduce the barriers to using cloud and distributed computing infrastructure to run complex, data-intensive analyses. The system has provided new methods for supporting research, including the ability to provide computing infrastructure on a virtual desktop via a web browser, run sophisticated analysis software (e.g. Marxan in Kepler workflow) and develop shareable workflows to support an ecosystem research and investigation framework. The ability to create reusable experiments as a set of interconnected tasks, and the ability to submit or invoke jobs on HPC, will make the concept of workflow very useful to promoting open science and bringing transparency to scientific experiments.

\section{Land Use and Land Resources Centre}

The increased analytical power available through the harnessing of cloud computing and high-performance processing (as well as enhanced models that blend data from multiple sources and work across spatial and temporal scales) provides an opportunity to take a more strategic approach to land use mapping and change monitoring. Coupled with the use of appropriate workflow approaches that enhance the ability to share, collaborate, automate, repeat and repurpose land use mapping systems, these advances present an opportunity to address the ongoing need for high-quality, scale-appropriate land use information products that are cost efficient and have high levels of precision, accuracy and currency. 
16. THE FUTURE FOR LAND USE MAPPING

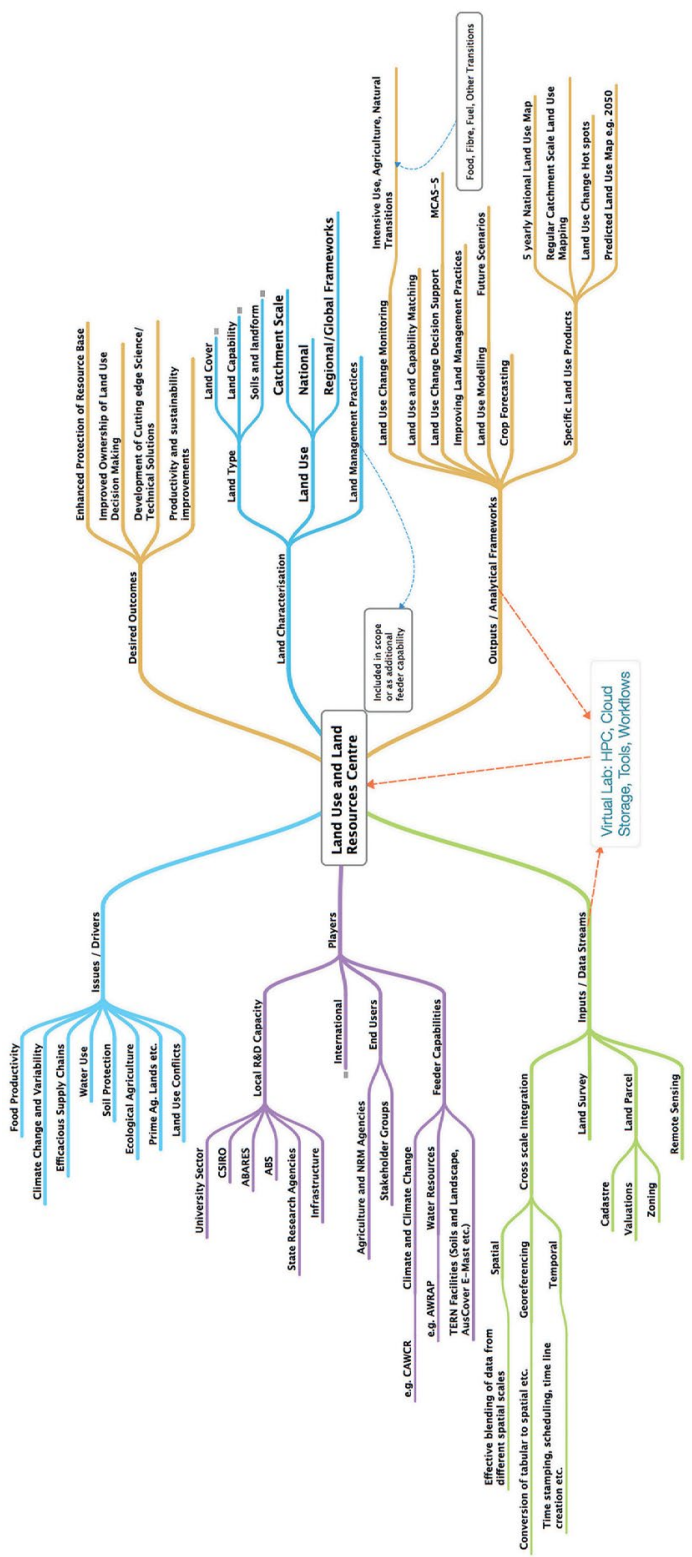

Figure 16.1: Organising framework for a land use and land resources centre designed to bring different capabilities together and leverage off existing investments by various stakeholders.

Note: This is a conceptual diagram. Moving clockwise from the top left: summary of 1) key drivers; 2) outcomes sought in policy and programs; 3) types of land resource layers required for analyses; 4) outputs the centre could produce; 5) required diversity of data to be accessed; and 6) stakeholders and collaborators needed. Source: Adapted from Clancy \& Lesslie (2013). 
The concept of a land use and land resources centre was scoped in recent times from a food security perspective (Clancy \& Lesslie, 2013). Beyond the central issue of food security, it was found that a broad range of needs could be addressed more directly and effectively by focusing specifically on improving Australia's capacity to analyse and track land use change, particularly in relation to our productive land assets. This would address stakeholder groups' demands, such as calls from the agricultural sector for improved data and analysis to assess land use change and agricultural production potential. A land use and land resources centre would provide an organising framework for bringing different capabilities together, while leveraging off existing investments (Figure 16.1).

The proposed land use and land resources centre would be a virtual organisation, operating on a collaborative basis and drawing on the expertise of Australian agencies and research institutions with allied interests and objectives. It would conduct an Australian program of work linked to international efforts on agricultural land use and food security analysis. In doing so, it could make an important contribution to global food security in terms of land use and land resources risk assessments, particularly the development of protocols for tracking and forecasting change, and the development of tools to assist decision-making.

\section{Recommendations}

1. The existing frameworks for national and state collaboration should be maintained and strengthened, including the direct involvement of science agencies such as the Commonwealth Scientific and Industrial Research Organisation (CSIRO), universities and the National Committee on Land Use and Management Information (NCLUMI).

2. It should be recognised that opportunities for harnessing HPC needs are central to discussions of national land use programs, including the NCLUMI.

3. It is necessary to move to a strategic approach that involves regular national and regional updates of land use mapping based on a consistent funding stream that recognises the broad range of uses and needs for land use products.

4. The concept of a land use and land resources centre should be further explored to address recommendations 1-3. 
5. If such a physical and virtual institution were to be established, naming it the (Rob) Lesslie Land Use and Land Resource Centre would be a fitting memorial to Rob's important work in this area.

\section{References}

Bryan, B. A. (2013). High-performance computing tools for the integrated assessment and modelling of social-ecological systems. Environmental Modelling and Software 39, 295-303. doi.org/10.1016/j.envsoft. 2012.02.006

Bryan, B. A., Crossman, N. D., King, D. \& Meyer, W. S. (2011). Landscape futures analysis: Assessing the impacts of environmental targets under alternative spatial policy options and future scenarios. Environmental Modelling and Software 26, 83-91. doi.org/10.1016/j. envsoft.2010.03.034

Clancy, T. F. \& Lesslie, R. G. (2013). A scoping assessment for a national research centre addressing land use and food security issues. Canberra, ACT: Department of Agriculture, Fisheries and Forestry.

Foley, J. A., DeFries, R., Asner, G. P., Barford, C., Bonan, G., Carpenter, S. R., ... Snyder, P. K. (2005). Global consequences of land use. Science 309(5734), 570-74. doi.org/10.1126/science.1111772

Francis, R. (2015, July). Research methods - the great integrator. Paper presented at the Australian eResearch Organisation's 6th National Forum: Roadmapping Australia's eResearch Future, Canberra. Retrieved from aero.edu.au/wp-content/uploads/2015/08/AeRO_ Forum_2015_Rhys_Francis_presentation.pdf

Guru, S. M., Nguyen, H. A., Banihit, S., Mulholland, M., Olsson, K. \& Clancy, T. (2015). Development of cloud-based virtual desktop environment for synthesis and analysis for ecosystem science community. International Workshop on Science Gateways, eResearch Australasia. Retrieved from eresearchau.files.wordpress.com/2015/11/guru_coesra _iwsg.pdf

Lesslie, R. \& Mewett, J. (2013). Land use and management: The Australian context (Research Report 13.1). Canberra, ACT: Australian Bureau of Agricultural and Resource Economics and Sciences. 
Ludäscher, B., Altintas, I., Berkley, C., Higgins, D., Jaeger, E., Jones, M., ... Zhao, Y. (2006). Scientific workflow management and the Kepler system. Concurrency and Computation: Practice and Experience 18, 1039-65. doi.org/10.1002/cpe.994

Zhao, G. D., Bryan, B. A., King, D., Luo, Z. K., Wang, E. L., BendeMichl, U., ... Yu, Q. (2015). Large-scale, high-resolution agricultural systems modelling using a hybrid approach combining grid computing and parallel processing. Environmental Modelling and Software 41, 231-38. doi.org/10.1016/j.envsoft.2012.08.007 
This text is taken from Land Use in Australia: Past, Present and Future, edited by Richard Thackway, published 2018 by ANU eView, The Australian National University, Canberra, Australia.

doi.org/10.22459/LUA.02.2018.16 